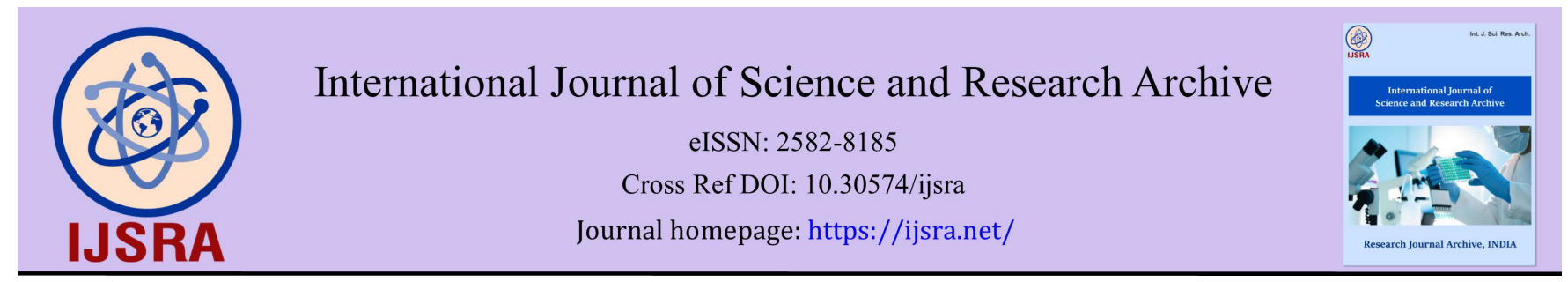

(RESEARCH ARTICLE)

Check for updates

\title{
Magnetic resonance imaging assessment of the cruciate ligament thickness among apparently healthy Nigerians
}

Kelechi Okwara ${ }^{1}$, Michael Sunday Okpaleke ${ }^{1,}{ }^{*}$, Uchenna Eusebius Dike ${ }^{2}$, Anthony Ugwu ${ }^{1}$, Joe Umunnah ${ }^{3}$ and Isiaq Jamiu 4

\author{
${ }^{1}$ Department of Radiography, Faulty of Health Sciences and Technology, College of Medicine, NnamdiAzikiwe University \\ Nnewi Campus, Nigeria. \\ 2 Department of Radiology, National Hospital Abuja, Nigeria. \\ ${ }^{3}$ Department of Medical Rehabilitation, Faulty of Health sciences and Technology, College of Medicine, NnamdiAzikiwe \\ University Nnewi Campus, Nigeria. \\ ${ }^{4}$ Department of Radiology, Lagos State University Teaching Hospital, Nigeria.
}

International Journal of Science and Research Archive, 2021, 03(02), 019-026

Publication history: Received on 18 July 2021; revised on 07 September 2021; accepted on 09 September 2021

Article DOI: https://doi.org/10.30574/ijsra.2021.3.2.0124

\begin{abstract}
Background: Anterior cruciate ligament (ACL) and posterior cruciate ligament (PCL) injuries are on the increase in Nigeria and there is no comprehensive study on the relationship between cruciate ligament thickness with age and gender using Magnetic Resonance Imaging (MRI) among Nigerians.
\end{abstract}

Aim of study: The study aimed to assess the thicknesses of the cruciate ligaments among apparently healthy Nigerian population using MRI.

Methods: A total of Ninety-four (94) volunteers aged 21 - 70 years who were referred for MRI knee assessment without any ACL and PCL injuries after radiological assessment of their MRI images by a consultant radiologist were recruited for the study. The study was carried out between June 2018 and December 2018. The Age and gender of the volunteers were recorded and the procedure was thoroughly explained to the participants. After removing all non - MRI compliant metallic objects from their bodies, the volunteers lied supine on the couch, arms by the sides, with head first and the knees were positioned on the knee coils and immobilized with cushions.The standard knee MRI examination protocol was adopted for the examinations using a 0.2 Tesla Siemens Magnetom Concerto MRI equipment. The ACL and PCL were measured at the femoral end, midline, and the tibia end using the computer electronic calipers, and the averages were calculated. The Age and gender - related differences in the cruciate ligament thicknesses were tested using Pearson's moment correlation coefficient and independent simple t-test respectively at a $5 \%$ level of significance.

Result: The average ACL thicknesses for male and female subjects were $4.76 \pm 0.78$ and $4.56 \pm 0.89$ respectively while the average PCL thicknesses for male and female subjects were $4.76 \pm 0.78$ and $4.75 \pm 0.79$ respectively. There were no statistically significant differences ( $p>0.005$ ) in mean values of ACL and PCL between male and female subjects respectively. PCL thicknesses increased with age while the ACL thicknesses decreased with age. Age correlated positively with the ACL( $r=0.2)$ as well with the PCL $(r=0.4)$.

Conclusion: The growths of the posterior and anterior cruciate ligaments have direct and inverse relationships with age respectively. Male volunteer subjects have thicker cruciate ligaments compared to female volunteer subjects.

\footnotetext{
${ }^{*}$ Corresponding author: Michael Sunday Okpaleke

Department of Radiography, Faulty of Health sciences and Technology, College of Medicine, Nnamdi Azikiwe University Nnewi Campus, Nigeria.

Copyright $(2021$ Author(s) retain the copyright of this article. This article is published under the terms of the Creative Commons Attribution Liscense 4.0.
} 
International Journal of Science and Research Archive, 2021, 03(02), 019-026

Keywords: Anterior cruciate ligament; Posterior cruciate ligament; MRI, Cruciate ligament thickness; Assessment

\section{Introduction}

\subsection{Background of the Study}

The anterior cruciate ligament (ACL) and posterior cruciate ligament (PCL) are intra-capsular, extra-synovial ligaments of the knee. They are important stabilizers of the knee and are named by the location of their tibial attachments [1]. Injuries to the ACL and PCL usually occur due to extreme hyperextension or twisting trauma such as a sports/physical injury, car crash or similar accident. The ligaments may rupture entirely as incomplete rupture of individual fiber may be torn as it occurs in partial cruciate ligament rupture. Partial ruptures of the cruciate ligaments are associated with the disruption of part of the ligament or the presence of a fluid signal in the ligament.

The use of stress X-rays, multi-detector computed tomography, and Ultrasonography may be useful as a screening tool for patients suspected to have cruciate ligament injury. They provide an objective measurement and determine the extent of an injury prior to the surgery and assess the level of healing in non-operative and postoperative conditions. However, magnetic resonance imaging (MRI) is most valuable for evaluating the acutely injured knee when repair of the cruciate ligament is considered since the clinical assessment of the ligaments can be difficult. In addition, MRI is non -invasive and provides detailed image structures within the knee joint including bones, cartilage, tendons, ligaments, muscles, and blood vessels, from many angles. On sagittal T2-weighted images, the normal ACL appears as a straight band parallel to the intercondylar roof. It fans out as it descends to its broad-based anterior tibial attachment. The ACL has a predominantly low signal on T1 or T2 weighted images. Some high signal striations are often seen within the distal ligament because of the interposition of fat or synovia. On sagittal T2-weighted images, the normal PCL appears as a well-defined uniform band of very low signal intensity. When the PCL is lax in extension, it has a gentle posterior convex curvature. A small round or oval structure of low signal intensity is often seen anterior or posterior to the PCL. It represents the menisco-femoral ligament, which extends obliquely from the posterior horn of the lateral meniscus to the medial femoral condyle.

Coronal and axial images are complementary in the evaluation of the femoral and tibial attachments of both cruciate ligaments. Overall, the ACL is higher in signal intensity than PCL [1].

Changes in the thickness of the anterior and posterior cruciate ligaments have been reported to be affected by age and gender using MRI[2]. The changes in thicknesses of these ligaments with respect to age and gender for Nigerians are unknown as racial differences tend to affect these ligaments' thickness [2,3]. Meniscal injuries associated with the ACL and PCL have been reported in different parts of Nigeria and the prevalence is on the increase $[3,4]$. Early identification and diagnosis of cruciate ligament pathologies will help clinicians and surgeons in assessing possibilities of anterior and posterior cruciate ligaments injuries based on changes in the thickness of the ligaments with age or gender for possible early management and reconstruction therapies and may provide guidance for greater precision and lower risk in choosing the thickness of the graft to be used in ligament reconstruction surgeries [5]

Various imaging modalities are used for the diagnosis of injuries associated with the ACL and PCL including multidetector computed tomography, ultrasound and MRI. While computed tomography imaging for the assessment of ACL or PCL is invasive, ultrasonography is believed to be a useful imaging tool for the visualization of the Ligaments [6]as well as measurement of the thickness of the ligaments [7] especially in situations where MRI facility is not available or cannot be done or isolated ACL or PCL injury is suspected but the most reliable non-invasive imaging modality is MRI ${ }^{1}$.

Previous studies in Hong Kong, China, and Singapore on the assessment of the cruciate ligament injuries of the knee, revealed the importance of MRI in the diagnosis of cruciate ligament injuries. The MRI signs of ACL tear include nonvisualization of the ACL, discontinuity of the ligament with abnormal increased T1 and T2 signals due to oedema and hemorrhage as well as thickening [1] Their work suggested that an anterior tibial translation greater than $5 \mathrm{~mm}$ is a helpful sign in the diagnosis of a complete ACL tear and they further explained that PCL is best evaluated on sagittal T2weighted images. A normal intact PCL is a well continuous band of low signal intensity in all pulse sequences. Discontinuous fibers with the fluid signals completely traversing the fiber, PCL thickening with increased signal intensity are some of the signs of PCL tear based on their findings. It was concluded that MRI is useful in the diagnosis of internal rearrangements of the knee and injuries of the cruciate ligaments [1].

Charles et al., (2017) [8] did a work titled "Posterior Cruciate Ligament Injury" where they revealed that PCL injuries are less common than ACL injuries and also that PCL is broader and stronger than ACL with PCL tensile strength as high as $2000 \mathrm{~N}$. Another study evaluated the shape of the native ACL along its length in relation to the PCL and compared it 
with the size of the three [3] commonly used autographs (bone-patellar tendon-bone), in their work entitled "The shape and the Thickness of the Anterior Cruciate Ligament along its Length in relation to the Posterior Cruciate Ligament". This research was carried out in Ioannina, Greece and they reported that the PCL occupies the largest part of the intercondylar area leaving only a small space for the ACL in the knee extension [7]. The ACL mid-substance has a width of $5 \mathrm{~mm}$, resembling a band shape only before its tibial insertion where the ACL fans out and takes the form of its tibial attachment. These dimensions of the native ACL have to be considered in graft selection for anatomic ACL reconstruction. Another study on the relationship between the thickness of the ACL, anthropometric variable and anatomical measurements on the knee using MRI in Brazil with an aim of ascertaining the thickness of the ACL and to ascertain whether there is an association between the variations in ligament thickness and patients' age and height[8] was carried out by Peterson et al.,(2017). They found out that the mean thickness of the middle third of the ACL to be $4.5 \mathrm{~mm}$ and $4.3 \mathrm{~mm}$ on sagittal and coronal planes respectively. The study also found no statistically significant relationship between the thickness of the ACL and the patients' age or height but a positive association with the size of the lateral femoral condyle and intercondylar distance were observed.

A more recent study revealed the relationship between age and the morphological characteristics of the ACL using MRI in a work titled 'Evaluation of age related differences in ACL size [9]. Thirty-seven young subjects who were diagnosed with menisci injury without ACL tear using MRI (15 male and 22 female, median age 26 years, range 21-49 years), and 33 elderly subjects for whom knee MRI was performed before uni-compartmental knee arthroplasty (11 male and 22 female, median age $=77$ years, range $=60-83$ years), were included in the study. In the elderly group, healthy ACL gross morphology was confirmed macroscopically during surgery. In all knees, ACL was detected without any intensity alteration. In the MRI evaluation, using the axial slice revealing the greatest length between the medial and lateral epicondyle of the femur, axial ACL size was evaluated. Using the coronal plane image and the sagittal image was sliced parallel with the native ACL. In the sagittal image of the MRI, the largest area of the ACL was measured. Statistical analysis was performed to reveal the correlation between age and ACL size. Both axial and sagittal ACL areas were compared between the young and elderly groups. The result showed that age and sagittal ACL area were significantly correlated. The sagittal ACL area was significantly larger in the young group when compared with the elderly group. In conclusion, for clinical relevance, sagittal ACL size was significantly larger in young subjects. The reason for this difference is likely the difference in knee size. Also another recent related study established the relationship between ACL growth with age in pediatric patients. The physical status, length of the ACL-tibial inclination angle, notch width index, the midpoint of the ACL tibial attachment, anterior and posterior dimensions of the tibia and tibial epiphyseal height were measured. Pearson's correlation coefficient was used to determine the strength of the association of each parameter relative to age. This demonstrated that ACL grows in diameter and length in a predictable fashion until age of 18 years [10]. These studies were done outside the shores of Nigeria and no study till date to the best of our knowledge has measured cruciate ligament thickness using MRI and established its relationship with anthropometric variables such as age and gender in Nigeria, hence the need for this study. Also, despite the geographical differences in cruciate ligament thickness, as well as the increasing prevalence of cruciate ligament injuries in Nigeria $[2,3,4]$, there is no comprehensive study on the relationship between cruciate ligament thickness with age and gender among Nigerians. The aim of this study was to assess the thicknesses of the cruciate ligaments among apparently healthy Nigerian population using MRI. The findings of this study will help clinicians in the evaluation of the status of cruciate ligaments for possible pathology based on the changes in its thickness for early detection of meniscal injuries, planning of possible surgical interventions and management.

\section{Material and methods}

This study adopted a prospective cross - sectional design. This study was carried out on volunteers who had MRI examination of the knee in the Radiology Department of National Hospital Abuja (NHA), Nigeria with no cruciate ligament injury. A Siemens Magneton Concerto Magnetic Resonance Imaging equipment (0.2 Tesla) with the Monitor and its measuring accessories were used for this study.

A total of Ninety-four (94) volunteers aged 21 - 70 years who were referred for MRI knee assessment without any ACL and PCL injury within the study period were enlisted in the study. Volunteers with ACL and PCL injuries were excluded from the study after expert radiological assessment of their knee MRI images by a consultant Radiologist. Also, volunteers who met the inclusion criteria and who were below 18 years of age were excluded from the study. The age and gender of the volunteers were recorded and the procedure was thoroughly explained to the participants to solicit their co-operation for the investigation. Claustrophobic subjects were supported by a relative or staff. 


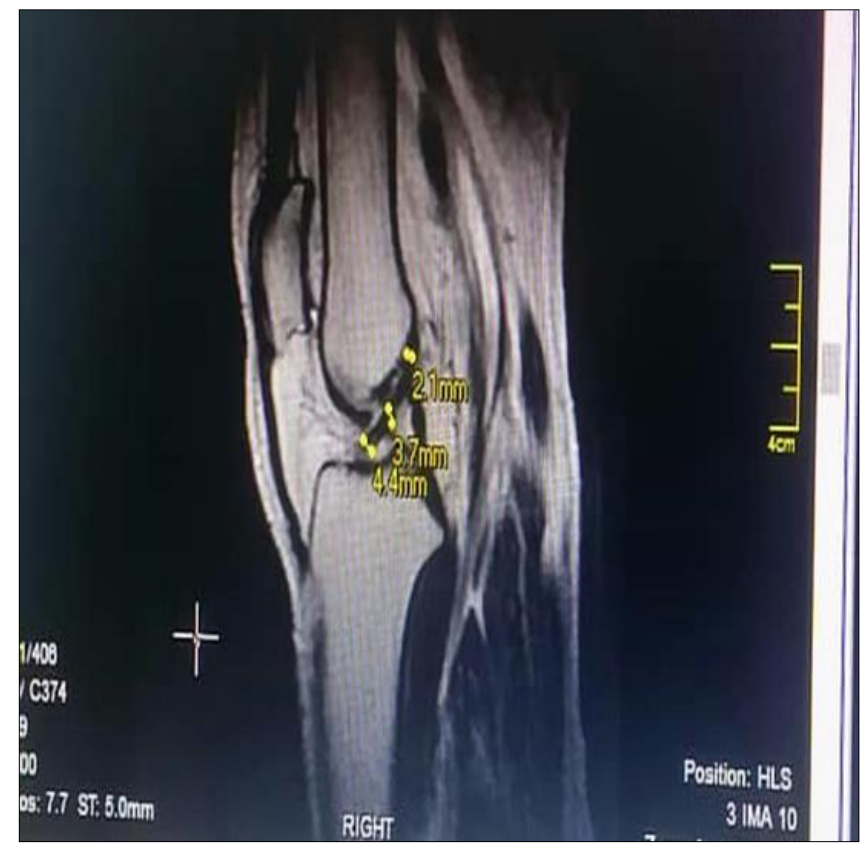

Figure 1 Measurement of ACL thickness on MR T2 saggital image

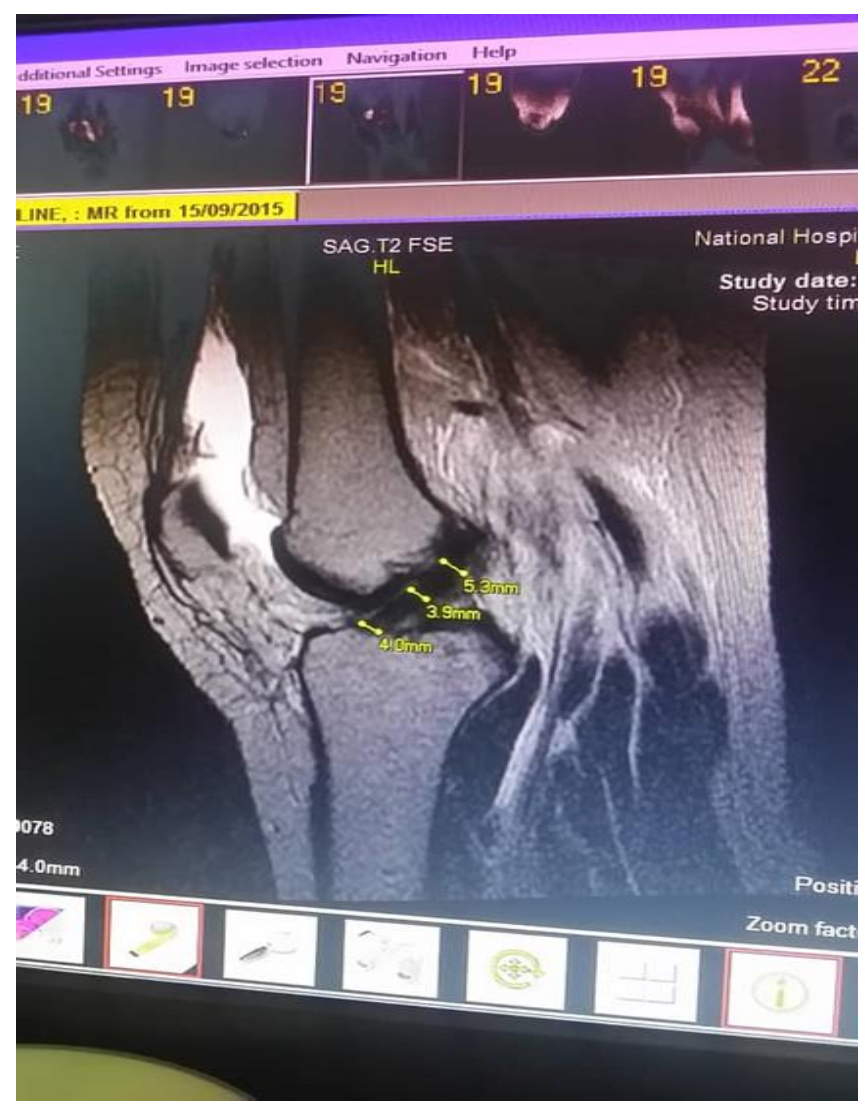

Figure 2 Measurement of the PCL thickness on MR T2 saggital image

The volunteers were asked to remove all metallic objects including keys, coins, wallet and cards with magnetic strips, jewelry, hearing aid, and hairpins. Patients lie supine on the couch, arms by the sides, with head first and the knees positioned on the knee coils and immobilize with cushions. The laser beam was centered over the lower border of the 
patella The standard knee MRI examination protocol was adopted for all subjects. The vertical gantry ray was centered $2.5 \mathrm{~cm}$ above the apex of the patella of the subject, while the horizontal ray was centered at the mid-lateral wall of the knee. A scan projection covered from the lower $1 / 3^{\text {rd }}$ of the femur to the upper $1 / 3^{\text {rd }}$ of the tibia. Image criteria included the visualization of the entire knee joints. The sagittal T2 weighted sequences were also acquired and all the images were reviewed by a consultant Radiologist to ensure that there were no ACL or PCL injuries. Thereafter, the thickness of the ACL and PCL were measured at the femoral end, midline and the tibia end using the computer electronic calipers and the averages were calculated.

\subsection{Data Analysis}

Data were first categorized based on gender and age groups. Both descriptive and inferential statistics were used to analyze the data using statistical package for social sciences (SPSS) version 23.0 (SPSS Inc. Chicago IL, USA) and statistical level of significance was set at $\mathrm{p}<0.05$.

\section{Results}

The sample population comprises of 45 males (48\%) and 49 (52\%) females. The ages of the subjects ranged from 21 years to 70 years (Table 1 ).

Table 1 Age distribution of the participants

\begin{tabular}{|l|c|c|}
\hline Age Group & Frequency & Percentage (\%) \\
\hline $21-30$ & 26 & 27.7 \\
\hline $31-40$ & 20 & 21.3 \\
\hline $41-50$ & 16 & 17.0 \\
\hline $51-60$ & 26 & 27.7 \\
\hline $61-70$ & 6 & 6.4 \\
\hline TOTAL & 94 & 100 \\
\hline
\end{tabular}

The mean age of females was 42 years, which is slightly higher than the mean age of male subjects which was 41 years.

The average thickness of the anterior ligament measurements decreased with age, while the average thickness of the posterior ligament measurements increased with age (Table 2).

Table 2 Average Cruciate Ligament thickness at different measurement sites with respect to age

\begin{tabular}{|c|c|c|c|c|c|c|}
\hline Age & Measuremen & s thickness & & & & \\
\hline $\begin{array}{c}\text { (years) } \\
\text { Mean } \pm S D \\
(\mathbf{m m})\end{array}$ & $\begin{array}{c}\text { A. F } \\
\text { Mean } \pm \text { SD } \\
(\mathbf{m m})\end{array}$ & $\begin{array}{c}\text { P. F } \\
\text { Mean } \pm \text { SD } \\
(\mathbf{m m})\end{array}$ & $\begin{array}{c}\text { A. Middle } \\
\text { Mean } \pm \text { SD } \\
(\mathrm{mm})\end{array}$ & $\begin{array}{c}\text { P. Middle } \\
\text { Mean } \pm \text { SD } \\
(\mathbf{m m})\end{array}$ & $\begin{array}{c}\text { A. T } \\
\text { Mean } \pm S D \\
(\mathrm{~mm})\end{array}$ & P. T \\
\hline $21-30$ & $5.54 \pm 0.59$ & $4.57 \pm 1.05$ & $4.90 \pm 0.71$ & $4.46 \pm 0.83$ & $5.74 \pm 0.67$ & $4.31 \pm 0.62$ \\
\hline $31-40$ & $5.18 \pm 0.80$ & $4.70 \pm 1.02$ & $4.84 \pm 0.92$ & $4.63 \pm 0.51$ & $5.54 \pm 1.63$ & $4.58 \pm 0.77$ \\
\hline $41-50$ & $4.90 \pm 0.65$ & $5.10 \pm 1.34$ & $4.63 \pm 0.79$ & $4.96 \pm 1.32$ & $5.20 \pm 1.13$ & $4.62 \pm 0.87$ \\
\hline $51-60$ & $4.74 \pm 0.89$ & $5.36 \pm 1.03$ & $4.32 \pm 0.85$ & $5.00 \pm 0.57$ & $4.20 \pm 0.94$ & $4.86 \pm 1.51$ \\
\hline $61-70$ & $4.60 \pm 0.71$ & $6.10 \pm 1.56$ & $4.23 \pm 0.65$ & $5.62 \pm 1.05$ & $4.00 \pm 0.65$ & $5.05 \pm 0.92$ \\
\hline Mean & $4.80 \pm 0.89$ & $4.95 \pm 1.01$ & $4.42 \pm 1.06$ & $4.63 \pm 0.96$ & $4.78 \pm 0.31$ & $4.70 \pm 0.99$ \\
\hline
\end{tabular}


The highest value $(6.10 \pm 1.56 \mathrm{~mm})$ was the average posterior cruciate ligament diameter at the femoral end among the respondents whose age ranges between 61 to 70 years, while the least value $(4.00 \pm 0.65 \mathrm{~mm})$ was recorded for anterior cruciate ligament thickness at the tibia for the respondents between the ages of 61 to 70 years (Table 2).

The mean values of diameters of ACL and PCL decreased and increased with age respectively (tables 2 and 3).

Table 3 Relationship between age and cruciate ligament mean diameters at the three measurement sites

\begin{tabular}{|l|c|c|}
\hline Age Group (Yrs.) & ACL mean \pm SD & PCL mean \pm SD \\
\hline $21-30$ & $5.39 \pm 0.66$ & $4.45 \pm 0.83$ \\
\hline $31-40$ & $5.19 \pm 1.12$ & $4.64 \pm 0.77$ \\
\hline $41-50$ & $4.91 \pm 0.86$ & $4.89 \pm 1.18$ \\
\hline $51-60$ & $4.42 \pm 0.89$ & $5.07 \pm 1.04$ \\
\hline $61-70$ & $4.28 \pm 0.67$ & $5.59 \pm 1.18$ \\
\hline
\end{tabular}

The highest average ACL and PCL were seen among age groups 21-30years (5.39 \pm 0.66$)$ and 61-70 years (5.59 \pm 1.18$)$ respectively while the least average ACL and PCL were seen among 61-70 (4.28 \pm 0.67$)$ and 21-30 (4.45 \pm 0.83$)$ respectively (table 3$)$

Male volunteer subjects have thicker cruciate ligaments for all the measurements (table 4). The highest cruciate ligament thickness for males were seen with the measurement of the posterior femur with the value of $4.97 \pm 0.92 \mathrm{~mm}$, while the least thickness for male respondents were seen with the measurement of the anterior middle with the value of $4.58 \pm 0.97 \mathrm{~mm}$. Similarly, the highest cruciate ligament thickness for female respondents were seen with the measurement of posterior femur with a value of $4.92 \pm 1.09 \mathrm{~mm}$, while the least thickness for female respondents were seen with the measurement of the anterior middle with the value of $4.55 \pm 1.13 \mathrm{~mm}$ (Table 4 ).

The anterior and posterior cruciate ligaments correlated positively with age. However, there is a weak positive correlation ( $\mathrm{r}=0.2$ ) between age and ACL diameters while there is a moderate positive correlation $(\mathrm{r}=0.42)$ between age and PCL diameters (Table 5).

Table 5 Correlation between age and cruciate ligament diameters

\begin{tabular}{|l|c|}
\hline \multicolumn{1}{|c|}{ Cruciate ligaments } & Age \\
\cline { 2 - 2 } & Person's moment correlation coefficient (r) \\
\hline Anterior cruciate ligament & 0.2 \\
\hline Posterior cruciate ligaments & 0.42 \\
\hline
\end{tabular}

The average ACL thickness for males and females were $4.76 \pm 0.78$ and $4.56 \pm 0.89$ respectively while the average PCL thickness for males and females were $4.76 \pm 0.78$ and $4.75 \pm 0.79$ respectively (table 6 ). $T$-test showed no statistical difference ( $p>0.005$ ) in mean values of ACL and PCL between male and female subjects respectively (Table 6).

Table 6 T-test of cruciate ligament diameters in Male and Female

\begin{tabular}{|l|c|c|}
\hline Gender & ACL Mean \pm SD & PCL Mean \pm SD \\
\hline Male & $4.76 \pm 0.78$ & $4.76 \pm 0.78$ \\
\hline Female & $4.56 \pm 0.89$ & $4.75 \pm 0.79$ \\
\hline p- value & 0.57 & 0.34 \\
\hline
\end{tabular}

\section{Discussion}

A total of ninety-four participants including 48\% $(n=45)$ males and $52 \%(n=49)$ females were enrolled for this study (table 1). The volunteers were grouped into five age groups at an interval of ten units to ensure adequate representation 
of subjects at each age group. The mean age of females was 42 years (range:21-70 years) which is slightly higher than the mean age of male participants which was 41years (range: 21-70 years) but there was no statistical difference in age between them.

This study revealed that the average cruciate ligament thickness increases and decreases with the age of the volunteer subjects. While the anterior cruciate ligaments decreased in thickness with increasing age, the posterior cruciate ligament increased proportionately in thickness with increasing age. The posterior cruciate ligament showed marked growth especially at the femoral end compared to the anterior cruciate ligaments. This is consistent with a related study that reported that ACL grows in thickness until the age of 18years [10]. The earlier study revealed that ACL thickness showed an average of $0.22 \mathrm{~mm}$ of growth per year between 1.5 and 18.75 years old with growth completed by age 18.75 years and subsequent decrease in diameter at a later age. Also, the increase of the posterior cruciate ligaments thickness with age in the present study is in agreement with the work of Peterson et al., (2017) [8] which stated that the PCL grows broader and stronger with age than ACL with higher tensile strength. It however accounts for why PCL injuries were less common than ACL injuries.

There were positive correlations between age and thickness of anterior and posterior cruciate ligaments. The thickness of the posterior cruciate ligament correlated better with age than the thickness of the anterior cruciate ligaments. This implies that age plays a significant role in the thickness of both the anterior cruciate ligament and the posterior cruciate ligament. In the present study, the anterior cruciate ligament was wider at young age than at old age thereby exhibiting an inverse relationship with age. However, the posterior cruciate ligaments thickness increased with age having a greater thickness at old age than at ayoung age. This finding is consistence with the findings of recent studies [9,10]. Infact, Iriuchishimaet al., [9] reported that 'the anterior cruciate ligament areas on the sagittal plane were significantly larger in the younger age group when compared to the elderly age group' similar to the findings of the present study. Although, this work showed a positive correlation between age and cruciate ligaments thickness, however, there was no statistically significant relationship $(P>0.05)$ between the average cruciate ligament's thicknesses across the age groups. This finding was in agreement with the findings of related studies which found out that age was not significantly associated with anterior cruciate ligament thicknesses $[2,8,11]$. The present study also revealed that the male gender has thicker cruciate ligaments than the female gender (table 4). However, further statistical analysis using $t-$ test showed no statistically significant difference in cruciate ligament thickness between male and female volunteer subjects. This is in agreement with the findings of similar studies by group of researchers that stated that men have wider ACL width than women [12] and there were no significant differences $(P>0.05)$ in the average thickness of cruciate ligaments between male and female subjects $[11,12]$ which were similar to the findings of the present study.

\section{Conclusion}

MRI assessment of the cruciate ligaments in apparently healthy volunteer subjects showed that the growths of posterior and anterior cruciate ligament have direct and inverse relationships with age respectively. Male volunteer subjects have thicker cruciate ligaments compared to female volunteer subjects.

\section{Compliance with ethical standards}

\section{Acknowledgments}

We acknowledge the staff and management of the National Hospital Abuja for their support in the conduct of this research.

\section{Disclosure of conflict of interest}

All the authors declare no conflict of interest.

\section{Statement of ethical approval}

Ethical approval for this study was given by the Health Ethics Research Committee of Federal Capital Territory Administration Abuja, Nigeria with approval number FHREC/2018/ 01/68/06-06-18.

\section{Statement of informed consent}

Informed consent was obtained from all the volunteers before the commencement of the study. 


\section{References}

[1] Kam C.K., Daniel W.Y. Chee, Wolfred C.G. Peh (2010) Magnetic Resonance Imaging of Cruciate Ligament Injuries of the Knee. Canadian Association of Radiologist Journal 61:80-89

[2] Victor Marques de Oliveira and Ricardo de Paul LeiteCury (2016) Study on the relationship between the thickness of the anterior cruciate ligament, anthropometric data and anatomical measurement of the knee. Revista Brasileira de Orthopedia 15(2): 194-199

[3] Laura M. Fayad, Elan H. Rosenthal, William B. Monisu, John A. Canino (2008) Anterior cruciate ligament volume analysis of gender difference. Journal of Magnetic Resonance Imaging 27(1): 218-223.

[4] Nkanta C.A., Alabi A.I.,Okoh N. (2017) Epidemilogy of meniscal injuries associated with anterior cruciate ligamenet (ACL) deficient knees in Northern Nigeria.Dala Journal of Orthopaedics (DJO) 2017; 1(1): 19-23

[5] Ndubuisi CC, Nnadozie UU, Anekwu EM, Maduba CC, Ekwedigwe HC, Ojukwu PC, Anyachukwu C, Ndubuisi G. Prevalence of anterior cruciate ligament injury among amateur footballers in Enugu, South-East Nigeria: The need for injury prevention programs. Niger J Med [serial online] 2020 [cited 2021 Jul 26];29:422-7. Available from: http://www.njmonline.org/text.asp?2020/29/3/422/295297.

[6] Palle Lalitha, M ChBalaji Reddy, K Jagannath Reddy, Vijaya Kumari (2010) Ultrasound evaluation of normal and abnormal posterior cruciate ligament-A prospective study. Pakistan Journal of Radiology 20(3): 125-129

[7] Pei-Tsen Chen, Wen-Shiang Chen (2013) Sonography of Normal Anterior Cruciate Ligament: A Preliminary Report. Journal of Medical Ultrasound 1(21): 16-20.

[8] Charlse S Peterson, Thomas Agesen, Jonos P Ertl, Gyorgy Kovacs (2017) Posterior Cruciate Ligament Injury. Sports Medicine 7(5).

[9] Iriuchishima T, Ryu K and Fu F.H. (2019). Evaluation of age-related differences in anterior cruciate ligament size.Knee Surgery Sports Traumatol Arthroscopy. 27(1):223-229

[10] Emily Niu and Danielle Putur (2019) ACL growth with age in pediatric patients: An MRI study:OrthopeadicTournal of sports medicine 7(3)

[11] Parag S. M, Prem C, Vidya C.N, Abhilash P.J. and Sheik A.H (2014) Smaller ACL Diameter is a predictor of subjects prone to Ligament injuries: An ultrasound study. Biomedical Research International vol 2015.http://dx.doi.org/10.1155/2015/845689.

[12] Lazar Stijak, Vodosava Radinjic, Valentina Nikolic, Zoram Blagoievic, Milan Aksic (2009) Correlation between the morphometric parameters of the anterior cruciate ligament and the intercondylar width: gender and age differences: Knee surgery, Sports Traumatology, Arthroscopy 17(7): 812-817 